\title{
SOME NEW IDENTITIES FOR LEGENDRE POLYNOMIALS*
}

\author{
BY \\ HARRY L. JOHNSON \\ Virginia Polytechnic Institute and State University
}

Introduction. Legendre polynomials $P_{n}(x)$ and Legendre functions have a long history of applications to the solution of boundary value problems in spherical domains, [1, 2]. For some time the author has been interested in the properties of a classical solution of the mixed boundary value problem

$$
\begin{aligned}
\nabla^{2} w(\rho, \phi) & =0, \quad 0 \leqslant \rho \leqslant R, 0 \leqslant \phi \leqslant \pi, \\
w_{\rho}(R, \phi)+h_{1} w(R, \phi) & =H_{1}(\phi), \quad 0 \leqslant \phi \leqslant \phi_{0}, \\
w_{\rho}(R, \phi)+h_{2} w(R, \phi) & =H_{2}(\phi), \quad \phi_{0} \leqslant \phi \leqslant \pi,
\end{aligned}
$$

where $h_{1}$ and $h_{2}$ are distinct non-negative constants. The analysis used to solve this boundary value problem has led to certain identities for sums of Legendre polynomials and other related functions. To the author's knowledge, only a special case of one of these identities is stated in the literature.

The format of this note is to state the identities and then give an outline of the steps used in their proof.

The identities. Let $P_{n}=P_{n}(x)$ denote the $n$th order Legendre polynomial. Let $\phi$ and $\theta$ be angles in the range $0 \leqslant \phi \leqslant \pi, 0 \leqslant \theta \leqslant \pi, x=\cos (\phi), y=\cos (\theta), \alpha=\min (\phi, \theta)$, $\beta=\max (\phi, \theta)$, and $K(\phi, \theta)=|\cos (\phi)-\cos (\theta)|^{1 / 2}$.

The identities are

$$
\begin{aligned}
& K_{0}(\theta, \phi)=\sum_{n=0}^{\infty} P_{n}(y) P_{n}(x)=\frac{1}{\pi}\left(\int_{0}^{\alpha} \frac{d s}{K(s, \theta) K(s, \phi)}\right), \\
& K_{1}(\theta, \phi)=\sum_{n=0}^{\infty} \frac{P_{n}(y) P_{n}(x)}{n+\frac{1}{2}}=\frac{2}{\pi} I(\alpha) I(\pi-\beta),
\end{aligned}
$$

where

$$
I(\alpha)=\int_{0}^{\pi / 2}\left(1-\sin ^{2}(\alpha / 2) \sin ^{2}(s)\right)^{-1 / 2} d s,
$$

* Received November 16, 1982. 


$$
\begin{aligned}
K_{2}(\theta, \phi) & =\sum_{n=0}^{\infty} \frac{P_{n}(y) P_{n}(x)}{\left(n+\frac{1}{2}\right)^{2}}=\frac{1}{2 \pi} \int_{\theta}^{\pi} \int_{\phi}^{\pi} \frac{(s+t-|s-t|) d t d s}{K(s, \theta) K(t, \phi)}, \\
K_{3}(\theta, \phi) & =\sum_{n=1}^{\infty} P_{n}(y) P_{n}^{\prime}(x) \\
& =\frac{1}{2 \pi} \int_{0}^{\theta} \frac{d s}{[K(s, \phi)]^{3} K(s, \theta)}, \quad \theta<\phi, \\
& -\frac{1}{2 \pi \sin ^{2}(\phi)} \int_{0}^{\phi} \frac{\left[\sin ^{2}(s)+K^{2}(\theta, s) K^{2}(\phi, s)\right] d s}{[K(\theta, s)]^{3} K(\phi, s)}, \quad \phi<\theta .
\end{aligned}
$$

The change of variable $\sigma=\left[\left(\tan ^{2}(\alpha / 2) \cot ^{2}(s / 2)-1\right)\right]^{1 / 2}$ transforms the integral on the right-hand side of (1) into

$$
K_{0}(\theta, \phi)=\frac{1}{\pi} \sec (\alpha / 2) \csc (\beta / 2) \int_{0}^{\infty}\left(1+\sigma^{2}\right)^{-1 / 2}\left(\omega^{2}+\sigma^{2}\right)^{-1 / 2} d \sigma
$$

where

$$
\omega^{2}=1-\tan ^{2}(\alpha / 2) \cot ^{2}(\beta / 2)
$$

Setting $\theta=\pi$ in (5) yields

$$
K_{0}(\pi, \phi)=\sum_{n=0}^{\infty}(-1)^{n} P_{n}(x)=\frac{1}{2} \sec (\phi / 2) .
$$

Setting $\theta=0$ yields

$$
K_{0}(0, \phi)=\sum_{n=0}^{\infty} P_{n}(x)=\frac{1}{2} \csc (\phi / 2) .
$$

Equation (7) appears in [2]. In addition, one can show that

$$
K_{0}(\theta, \phi)=(1 / \pi) \sec (\alpha / 2) \csc (\beta / 2) \ln \left(1 / \omega^{2}\right)+k_{0}(\theta, \phi)
$$

where $k_{0}(\theta, \phi)$ is continuous on the rectangle $0 \leqslant \theta \leqslant \pi, 0 \leqslant \phi \leqslant \pi$, and $\omega^{2}$ is defined by (6).

Proof of identities. We give an outline of the proofs of the identities (1)-(4). To prove (1), write $P_{n}(x)$ and $P_{n}(y)$ in their Laplace integral form

$$
\begin{aligned}
& P_{n}(x)=P_{n}(\cos (\phi))=\frac{1}{\pi} \int_{0}^{\pi} z^{n} d \sigma, \\
& P_{n}(y)=P_{n}(\cos (\theta))=\frac{1}{\pi} \int_{0}^{\pi} \delta^{n} d t
\end{aligned}
$$

where $z=z(\phi, \sigma)=\cos (\phi)+i \sin (\phi) \cos (\sigma)$ and $\delta=\delta(\theta, t)=\cos (\theta)+i \sin (\theta) \cos (t)$. One can justify the interchange of the above integrations and the infinite series to obtain a geometric series in the variable $z \delta$. It is easy to show that 


$$
\frac{1}{\pi} \int_{0}^{\pi} \frac{d t}{(1-z \delta)}=k(z, \theta)=\left[\left(1-z e^{i \theta}\right)\left(1-z e^{-i \theta}\right)\right]^{-1 / 2} .
$$

Without loss of generality one may assume that $\phi<\theta$. In the last remaining integral the integration variable can be changed from $\sigma$ to the complex variable $z=\cos (\phi)+$ $i \sin (\phi) \cos (\sigma)$. This gives

$$
K_{0}(\theta, \phi)=\frac{1}{\pi i} \int_{C_{1}} k(z, \theta) k(z, \phi) d z
$$

where $C_{1}=\{z: z=\cos (\phi)+i y,|y| \leqslant \sin (\phi)\}$. The integrand in (9) is an analytic function of $z$ in the region $R=\left\{z: z=\rho e^{i s}, \cos (\phi) \sec (s) \leqslant \rho<1,|s|<\phi\right\}$; hence the line integral (9) can be transferred to a line integral over the arc $C=\left\{z: z=e^{i s},|s| \leqslant \phi\right\}$. Inserting this into (9) yields (1).

To prove (2), we again assume without loss of generality that $\phi<\theta$. The Legendre polynomials are represented by the Mehler's formulas

$$
P_{n}(x)=P_{n}(\cos (\phi))=\frac{2^{1 / 2}}{\pi} \int_{0}^{\phi} \frac{\cos \left[\left(n+\frac{1}{2} s\right)\right] d s}{K(s, \phi)}
$$

and

$$
P_{n}(y)=P_{n}(\cos (\theta))=\frac{2^{1 / 2}}{\pi} \int_{\theta}^{\pi} \frac{\sin \left[\left(n+\frac{1}{2}\right) t\right] d t}{K(\theta, t)} .
$$

This leads one to consider the partial sums

$$
\sum_{n=0}^{N} \frac{2 \cos \left[\left(n+\frac{1}{2}\right) s\right] \sin \left[\left(n+\frac{1}{2}\right) t\right]}{n+\frac{1}{2}}=\int_{0}^{s-t} S_{n}(\sigma) d \sigma+\int_{0}^{s+t} S_{N}(\sigma) d \sigma
$$

where

$$
S_{n}(\sigma)=\sum_{n=0}^{N} \cos \left[\left(n+\frac{1}{2}\right) \sigma\right]=\sin \left[\left(n+\frac{1}{2}\right) \sigma\right] / 2 \sin (\sigma / 2) .
$$

Using the Dirichlet theorem,

$$
\lim _{\omega \rightarrow \infty} \frac{2}{\pi} \int_{0}^{a} f(x) \frac{\sin (\omega x)}{x} d x=f(0+),
$$

one obtains, after some simple integrations, formula (2).

Equation (3) is obtained in a way very similar to that used to obtain (2). To obtain (4), one differentiates (8) with respect to $\phi$ and uses steps similar to those used to obtain (1).

\section{REFERENCES}

[1] E. W. Hobson, The theory of spherical and ellipsoidal harmonics, Chelsea Publishing Co., New York, 1955

[2] T. M. MacRobert, Spherical harmonics, third edition, Pergamon Press, Oxford and New York, 1967 